\title{
A New Method for the Registration of Mammograms
}

\author{
Yih-Chih Chiou ${ }^{1}$ Cheng-Yu Lin ${ }^{1}$ Yu-Teng Liang ${ }^{1,2}$ \\ ${ }^{1}$ Department of Mechanical Engineering, Chung Hua University \\ No. 707, Sec.2, WuFu Rd., Hsinchu, Taiwan 300, R.O.C. \\ ${ }^{2}$ Automation Engineering Department, Ta Hwa Institute of Technology \\ No.1, Dahua Rd., Qionglin Shiang Hsinchu Taiwan 307, R.O.C.
}

\begin{abstract}
Mammogram registration is a critical step in automatic detection of breast cancer. Much research has been devoted to registering mammograms using either feature-matching or similarity measure. However, few studies have been done on combining these two methods. In this research, a hybrid mammogram registration method is developed by combining feature-based and intensity-based image registration techniques. Besides, internal and external features were used simultaneously during registration to obtain a global spatial transformation. The experimental results indicate that the similarity between the two mammograms increases significantly after a proper registration using the proposed registration procedures.
\end{abstract}

Keywords: Mutual Information, Mammogram Registration, Thin-Plate Splines, Feature Matching

\section{Introduction}

Although many new technologies keep evolving, mammography remains the most effective and popular screening method for the early detection of breast cancer. As to the study of breast cancers, most computer-assisted diagnosis (CAD) programs attempt to detect anomalies in a single mammogram. However, in clinical practices, a so-called comparative analysis (CA) method is widely used. CA is helpful to the detection of anomalies. In practice, it is difficult to prevent breast from being displaced or deformed in response to the applied forces during the acquisition of the mammogram. As a result, the differences in shapes or positions might exist between two mammograms acquired in sequence. Accordingly, prior to $\mathrm{CA}$, it is necessary to minimize the discrepancies using image registration.

Image registration, a process to bring two images into alignment, is an important ongoing research topic $[1,2]$. In general, registration algorithms can be roughly divided into two main categories, i.e. feature-based and intensity-based registration methods. As mentioned earlier, CA methods are widely used in clinical practices. Hence, the current trend of CAD methods is toward comparing breast images from either the same modality or different modalities. Breasts are elastic bodies, thus all researches based on the presumption that takes breasts as rigid bodies are basically inappropriate [3,4]. As a result, many non-rigid-based mammogram registration techniques were proposed [5,6]. Although much work has been done on mammogram registration using either feature-based methods $[3,4]$ or intensity-based method $[7,8]$, few studies have been done on combining the aforementioned two techniques. In this research, a hybrid mammogram registration algorithm, which used both methods to bring mammograms into alignment, has been developed. In addition, the developed registration scheme used Thin-Plate Splines (TPS) spatial transformation theory to take into account the elastic deformations nature of breasts in response to pressure.

\section{Mammogram Registration Scheme}

Mammogram registration is a critical step in automated detection of breast cancer. In this paper, we use image preprocessing, mutual information, feature extraction, feature matching, similarity measure, and TPS coordinate transformation to bring two corresponding mammograms into registration. Figure 1 show the flow chart of the proposed registration algorithm.

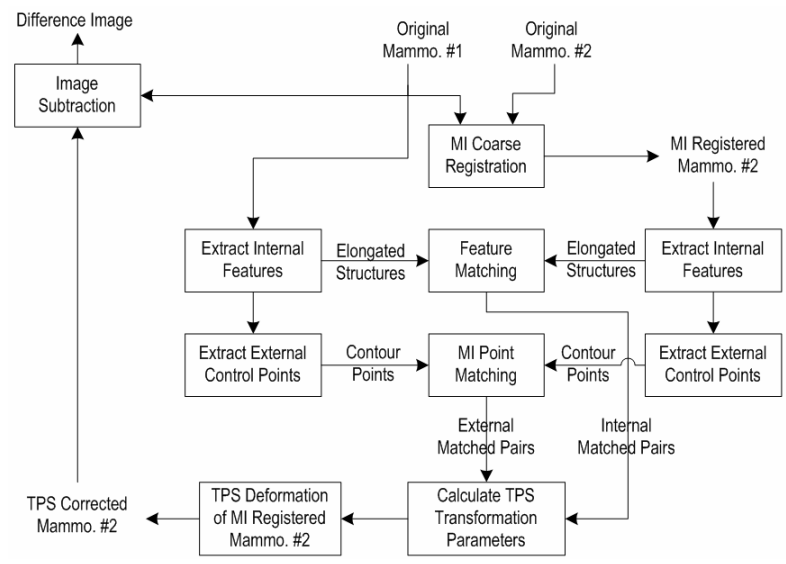

Fig. 1 Flow chart of the proposed algorithm for the registration of mammograms. 


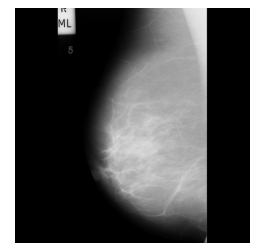

(a)

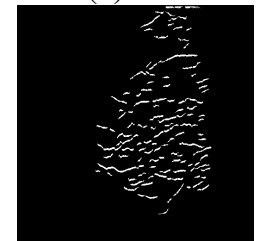

(d)

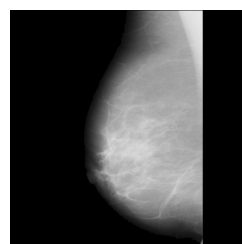

(b)

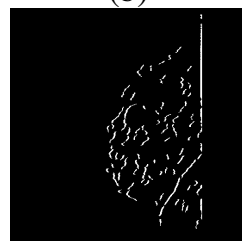

(e)

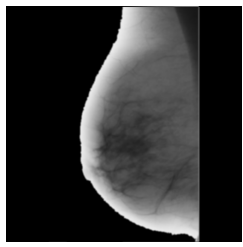

(c)

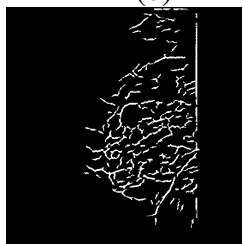

(f)
Fig. 2 Extraction of elongated structures: (a) mdb014; (b) segmented result of Fig. 2a; (c) inversed and Gaussian filtered result of Fig. 2b; (d) and (e) show the horizontal and vertical elongated structures extracted from Fig. 2c, respectively; (f) the ORing results of Figs. $2 \mathrm{~d}$ and $2 \mathrm{e}$.

\subsection{Coarse Registration}

Mutual information (MI), one of intensity-based registration methods, was commonly used to solve multimodality medical image registration problems $[9,10]$. The purpose of the coarse registration using MI is to reduce global spatial differences between corresponding mammograms caused by the positioning difference of the breasts during their acquisition. By finding a set of parameters, $\Delta x, \Delta y$, and $\Delta \theta$, capable of maximizing the MI between the two mammograms, the best registration location is found. This is indeed an unnecessary step for registration using TPS mapping algorithm; however it is beneficial to feature matching. More specifically, after MI coarse registration we can reasonably presume that the relative displacements of the corresponding control points have been minimized.

\subsection{Extraction of Internal Features}

Two types of features can be identified in a mediolateral mammogram: external features and internal features. External features include skin boundary and nipple. Internal features include pectoral muscles, glandular tissues, fatty tissues, and blood vessels. To extract internal features, we adopt similar procedures as suggested by Vujovic and Brzakovic [4]. The objective can be accomplished by first segmenting the breast region, then filtering the noises, and finally extracting the elongated structures. The detailed is described as follows.

2.2.1. Segmenting and Filtering. As shown in Fig. 2a, there exists an artificial label in the mammogram for identification purpose. To avoid mismatching of features caused by the label, breast regions were extracted from the mammograms by using segmenting and size-filtering. The resulting image is shown in Fig. 2b. Fig. 2c shows the inverted and Gaussian filtered mammogram.

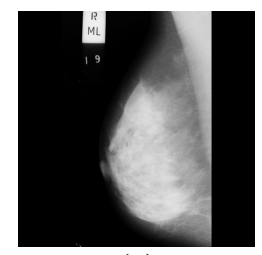

(a)

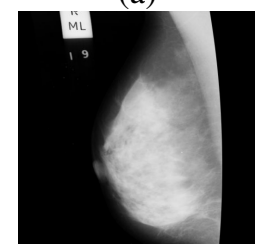

(d)

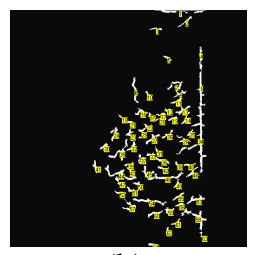

(b)

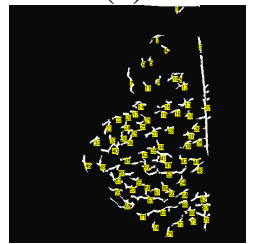

(e)

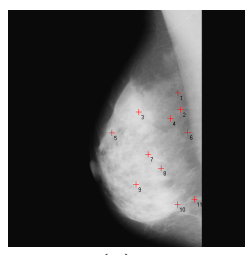

(c)

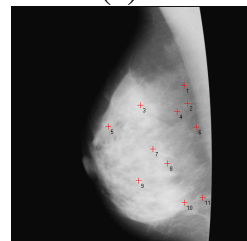

(f)
Fig. 3 Internal control points extraction and matching results: (a) and (d) denote the mdb004 and its simulated temporal counterpart; (b) and (e) show the features extracted from Figs. 3a and 3d, respectively; (c) and (f) show the corresponding matched points superimposed on the original mammograms Fig. 3a and Fig. 3b, respectively.

2.2.2. Elongated structure extraction. Horizontal and vertical elongated structures were extracted by using the horizontal and vertical types of MMO (Modified Monotony Operator). By convolving the image as shown in Fig. 2c with horizontal and vertical MMOs, we can obtain horizontal and vertical elongated structures as shown in Figs. 2d and 2e, respectively. Then by ORing Figs. $2 \mathrm{~d}$ and $2 \mathrm{e}$, we can obtain an image containing elongated structures in any direction as shown in Fig. $2 \mathrm{f}$. The elongated structures are indeed the objects that we are going to extract features from them. Readers who are interesting in the detailed operation of MMO can refer to reference [4].

\subsection{Feature Matching}

The key to the success of landmark-based registration is to establish a correct correspondence between objects extracted separately from a pair of mammograms. Before matching two elongated structures, each object's features, including area, perimeter, centroid, maximum diameter, minimum diameter, maximum diametric angle, and minimum diametric angle are calculated. To be a valid matched pair, the corresponding objects must meet all of the following criteria:

- Area ratio, perimeter ratio, and maximum diametric ratio are all within 0.8 and 1.2.

- Centroid distance is less than 20 pixels.

- Maximum diametric difference is less than 20 pixels.

- Minimum diametric difference is less than 15 pixels.

- Maximum diametric angle must be equal to the minimum diametric angle.

Refer to Figs. 3b and 3e, 63 and 75 elongated structures (yellow-colored blocks) were extracted from mdb004 (Fig. 3a) and its simulated counterpart (Fig. $3 \mathrm{~d}$ ), respectively. In spite of this, only 11 matched pairs (indicated by red-colored crosses as shown in Figs. $3 \mathrm{c}$ and $3 \mathrm{f}$ ) were obtained after feature matching. 


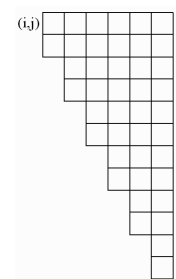

(a)

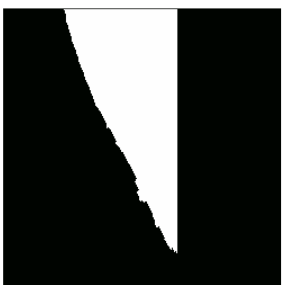

(b)

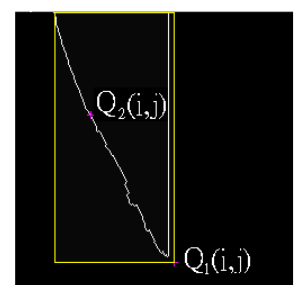

(c)
Fig. 4 Control points located on the interface of pectoral muscle and breast: (a) wedge-shaped mask of size 6 for extracting pectoral muscle region. (b) segmented pectoral muscle region; (c) designated control points $Q_{1}$ and $Q_{2}$.

\subsection{Designation of External Control Points}

Occasionally, imperfect registrations occur because few matched pairs are available, or the matched pairs are concentrated in a local area. To prevent the problems from occurring, we incorporate additional feature points obtained from breast contour and the B-P boundary (the boundary separating the breast and the pectoral muscle.

\subsubsection{Designate control points on pectoral muscle} region. Before designating control points on the $B-P$ boundary of the first mammogram, the wedge-shaped mask shown in Fig. 4a is used to segment the pectoral muscle regions from the first mammogram. Once the pectoral muscle region (Fig. $4 \mathrm{~b}$ ) has been extracted, its minimum enclosing rectangle can be obtained. The lower-right point of the rectangle is then denoted as the first control point, $Q_{1}$ having coordinates of $\left(i_{1}, j_{1}\right)$, as shown in Fig. 4c. After that, a second control point $Q_{2}$ located on the B-P boundary and at a distance of $S$ from $Q_{1}$ is specified. The choice of $S$ is based on the y-coordinate of $Q_{1}$.

2.4.2 Designate control points on breast contour. Before assigning control points on the breast contour of the first mammogram, we first obtain breast region using segmentation. Next, Laplacian edge detector is used to derive the breast contour. Finally, points, each at a distance of 80 pixels from the previous one, are designated along the breast contour.

\subsection{Point Matching}

Once the control points in the first mammogram have been assigned, their corresponding points in the second mammogram are located using mutual information. Use $Q_{l}$ as an example. In order to locate its corresponding point $Q_{1}^{\prime}$ in the second mammogram, an $m \times m$ region centered at $\left(i_{1}, j_{1}\right)$ of the first mammogram is defined as the feature region. For each control point, a rectangle region, called "feature zone", of certain size centered at each designated control point is created. Next, search the corresponding region in the second mammogram for $Q_{1}^{\prime}$ using mutual information. The searching is confined in an $n \times n$ region centered at $\left(i_{1}, j_{l}\right)$ of the second mammogram. Figure 5 shows the external matched pairs extracted from both mammograms.

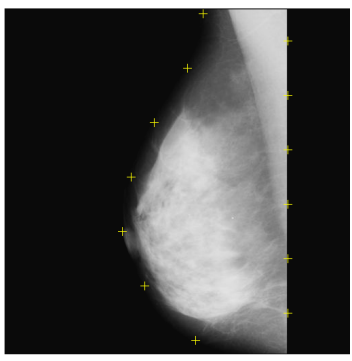

(a)

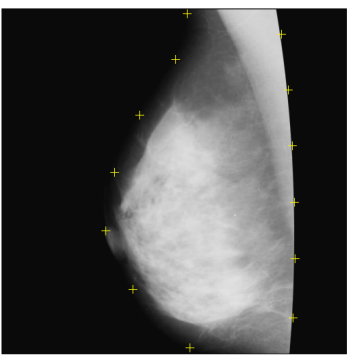

(b)
Fig. 5 MI point matching results: (a) the yellow-colored crosses denote the control points automated assigned in the first mammogram; (b) the corresponding control points located in its TPS simulated counterpart.

\subsection{TPS Transformation and Deformation}

The object of the current step is first to determine the spatial relationship between two images, and then use the derived spatial transformation matrix to transform one of the image in order that the two images will be perfectly registered. In this research, a radial basis TPS is applied to simulate the deformation of breasts. By using the matched pairs obtained during feature matching and MI point matching, we can obtain the spatial transformation matrix relating the two mammograms to be matched. And, the deformations of the mammogram to be registered can be corrected accordingly.

\section{Experimental Results and Discussions}

In this research, we used three approaches to derive transformation parameters, i.e., internal matched pairs only, external matched pairs only, and both internal and external matched pair. The mammograms were obtained from the MIAS digital mammogram database. The registration performance is evaluated in terms of $\mathrm{CC}$ and SSD of the two mammograms. Fig. 6 shows the registration results of two temporal mammogram pairs using the proposed registration algorithm. It is clear that most of the simulated deformations have been effectively recovered, as shown in Fig. 6d. TPS-registered (use the proposed technique to register) results are compared with the results of pre-registered (without registration). As shown in Table 1, the CC values for the pre-registered mammograms mdb007 and mdb016 are 0.900 and 0.888 , respectively. After TPS registration, the $\mathrm{CC}$ values rise to 0.997 and 0.990 , respectively. On the other hand, the SSD values decreased from 0.060 and 0.063 (pre-registered) to 0.010 and 0.019 (TPS-registered), respectively.

Table 1. Comparison of the pre-registered and TPS-registered results of temporal mammograms in terms of CC and SSD.

\begin{tabular}{ccccc}
\hline \multirow{2}{*}{ Mammogram } & \multicolumn{2}{c}{ Pre-registered } & \multicolumn{2}{c}{ TPS-registered } \\
\cline { 2 - 5 } & $\mathrm{CC}$ & $\mathrm{SSD}$ & $\mathrm{CC}$ & $\mathrm{SSD}$ \\
\hline 007 & 0.900 & 0.060 & 0.997 & 0.010 \\
\hline 016 & 0.888 & 0.063 & 0.990 & 0.019 \\
\hline
\end{tabular}


Table 2. Comparison of registration results using internal, external, or both internal and external feature points in terms of CC and SSD.

\begin{tabular}{|c|c|c|c|c|c|c|}
\hline \multirow[t]{2}{*}{ Mammog. } & \multicolumn{2}{|c|}{$\begin{array}{l}\text { External } \\
\text { Features }\end{array}$} & \multicolumn{2}{|c|}{$\begin{array}{c}\text { Internal } \\
\text { Features }\end{array}$} & \multicolumn{2}{|c|}{$\begin{array}{l}\text { Int. and Ext. } \\
\text { Features. }\end{array}$} \\
\hline & $\mathrm{CC}$ & SSD & $\mathrm{CC}$ & SSD & $\mathrm{CC}$ & SSD \\
\hline 007 & 0.973 & 0.029 & 0.994 & 0.012 & 0.997 & 0.010 \\
\hline 016 & 0.977 & 0.026 & 0.987 & 0.002 & 0.990 & 0.019 \\
\hline
\end{tabular}

Fig. 7 and Table 2 show the comparison results of using internal, external, or both internal and external feature points to register corresponding mammograms. As shown, if both internal and external feature points were used simultaneously, better registration accuracy can be obtained. The results suggest that both internal and external features should be used in registration if possible.

\section{Conclusions}

Strictly speaking, due to the deformable, inhomogeneous, and anisotropic natures of the breasts as well as the variations in the acquisition conditions, accurate mammogram registration is an intractable problem. However, in the paper, we have presented an innovative algorithm for the automatic registration of mammograms. The proposed algorithm uses a feature-based registration method to match internal features and an intensity-based registration method to match external features. Furthermore, by incorporating both intrinsic and extrinsic control points, the hybrid registration method do effectively solve the imperfect registration problems caused by few control points or non-uniform distribution of control points.

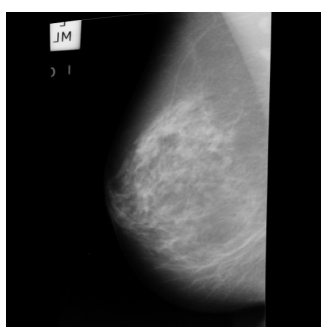

(a)

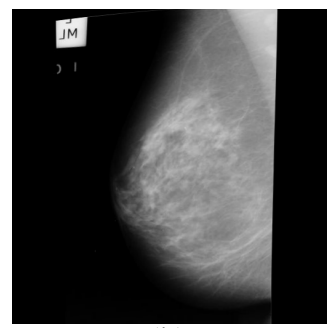

(b)

\section{References}

[1] Brown LG, "A Survey of Image Registration Techniques," ACM Computing Surveys, Vol.24, No.4, pp.325-376, 1992.

[2] Zitová B, Flusser J, "Image Registration Methods: A Survey," Image and Vision Computing, Vol. 21, Issue 11, pp.977-1000, 2003.

[3] Stamatakis EA, Ricketts IW, Cairns AY, Walker C, Preece PE, "Detecting Abnormalities on Mammograms by Bilateral Comparison, IEE Colloquium on Digital Mammography," London, UK, Mar. 27, 1996, pp.12/1-12/4.

[4] Vujovic N, Brzakovic D, "Establishing the Correspondence between Control Points in Pairs of Mammographic Images," IEEE Trans. Image Processing, Vol.6, No. 10, pp.1388-1399, 1997.

[5] Kostelec PJ, Weaver JB, Healy DM, "Multiresolution Elastic Image Registration," Medical Physics, Vol.25, No.9, pp.1593-1604, 1998.

[6] Sallam MY, Bowyer KW, "Registration and Difference Analysis of Corresponding Mammogram Images," Medical Image Analysis, Vol.3, No.2, pp. 103-118, 1999.

[7] Sivaramakrishna R, "Breast Image Registration Using a Textural Transformation," Computer Systems Engr., Medical Physics, Vol. 25, No.11, pp.2249, 1998.

[8] Wirth MA, Narhan J, Gray D, "Nonrigid Mammogram Registration Using Mutual Information," Proc. SPIE Medical Imaging: Image Processing, San Diego USA, February 2002, Vol.4684, pp.562-573.

[9] Maes F, Collignon A, Vandermeulen D, Marchal G, Suetens R, "Multimodality Image Registration by Maximization of Mutual Information," IEEE Trans. Med. Imag., Vol.16, No.2, pp.187-198, 1997.

[10] Pluim JPW, Maintz JBA, Viergerver MA, "Mutual-Information-Based Registration of Medical Images: A Survey," IEEE Trans. Med. Imag., Vol.22, No.8, pp.986-1004, 2003.

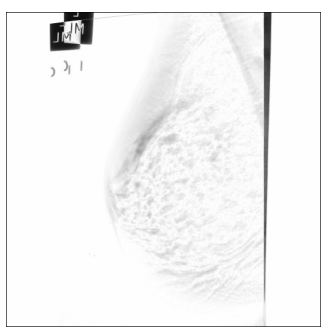

(c)

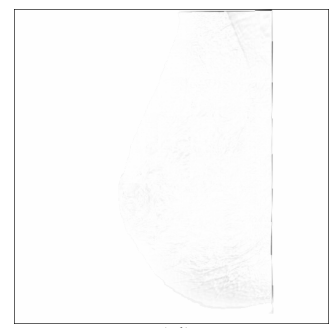

(d)

Fig. 6 Temporal mammogram registration results: (a) mdb007; (b) Simulated counterparts of Fig. 6a; (c) Difference images of Figs. 6a and 6b; (d) Difference images of Fig. 6a and its TPS corrected counterparts (not shown).

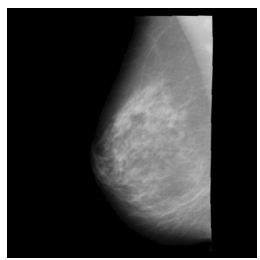

(a)

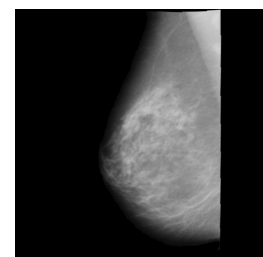

(b)

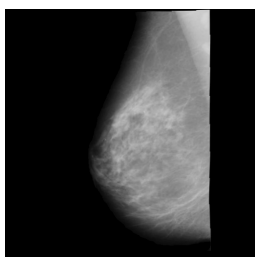

(c)

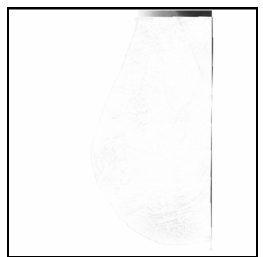

(d)

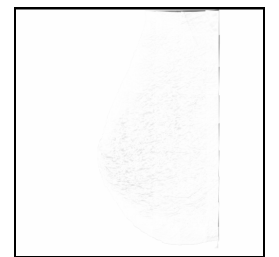

(e)

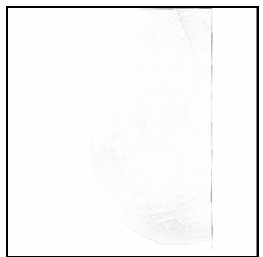

(f)

Fig. 7 Comparison of registration results: (a), (b), (c) show the TPS corrected mdb007 using external features, internal features, or external and internal features, respectively;.(d), (e), and (f) show the image subtraction results of (Fig. 6a-Fig. 7a), (Fig. 6a-Fig. 7b), and (Fig. 6a - Fig. 7c), respectively. 